\title{
STAR PARTIAL ORDER-HEREDITARY SUBSPACES IN $\mathscr{B}(\mathscr{H})$
}

\author{
QIAN ZHANG AND GUOXING Ji
}

\begin{abstract}
Let $\mathscr{B}(\mathscr{H})$ be the algebra of all bounded linear operators on a complex Hilbert space $\mathscr{H}$. It is proved that a weak operator topology closed nonzero subspace $\mathscr{M}$ in $\mathscr{B}(\mathscr{H})$ is hereditary with respect to the star partial order, that is, for any $A \in \mathscr{B}(\mathscr{H})$ and $B \in \mathscr{M}, A \in \mathscr{M}$ whenever $A^{*} A=A^{*} B$ and $A A^{*}=B A^{*}$, if and only if there is a unique pair of nonzero projections $P$ and $Q$ in $\mathscr{B}(\mathscr{H})$ such that $\mathscr{M}=P \mathscr{B}(\mathscr{H}) Q$.
\end{abstract}

Mathematics subject classification (2010): 06A06, 47A99.

Keywords and phrases: Star partial order, projection, hereditary subspace.

\section{REFERENCES}

[1] J. Antezana, C. Cano, I. Mosconi, D. Stojanoff, A note on the star order in Hilbert spaces, Linear and Multilinear Algebra, 58 (2010), 1037-1051.

[2] J. K. BAKSAlary, S. K. Mitra, Left-star and right-star partial orderings, Linear Algebra and its Applications, 149 (1991), 73-89.

[3] J. K. BAKSAlary, F. Pukelsheim, On the Löwner, minus and star partial ordering of nonnegative definite matrices and their squares, Linear Algebra and its Applications, 151 (1991), 135-141.

[4] J. B. Conway, A Course in Operator Theory, American Mathematical Society, Rhode Island, 2000.

[5] G. Dolinar, J. Marovt, Star partial order on $\mathscr{B}(\mathscr{H})$, Linear Algebra and its Applications, 434 (2011), 319-326.

[6] M. P. DRAZIN, Natural structures on semigroups with involution, Bulletin of the American Mathematical Society, 84 (1987), 139-141.

[7] J. GROSS, Löwner partial ordering and space preordering of Hermitian non-negative definite matrices, Linear Algebra and its Applications, 326 (2001), 215-223.

[8] R. E. HARTWIG, M. P. DRAZIN, Lattice properties of the star order for complex matrices, Journal of Mathematical Analysis and Applications, 86 (1982), 539-578.

[9] C. Pearcy, D. Topping, Sums of small numbers of idempotents, Michigan Mathematical Journal, 14 (1967), 453-465.

[10] P. ŚEmL, Automorphisms of $\mathscr{B}(\mathscr{H})$ with respect to minus partial order, Journal of Mathematical Analysis and Applications, 369 (2010), 205-213. 